\title{
Relations between social comparisons and physical activity among women in midlife with elevated risk for cardiovascular disease: an ecological momentary assessment study
}

\author{
Danielle Arigo ${ }^{1,2}$ (D) Jacqueline A. Mogle ${ }^{3}$. \\ Joshua M. Smyth ${ }^{4}$
}

Received: 17 March 2021 / Accepted: 23 April 2021 / Published online: 12 May 2021

(C) The Author(s), under exclusive licence to Springer Science+Business Media, LLC, part of Springer Nature 2021

\begin{abstract}
Women in midlife (ages 40-60) show decreases in physical activity (PA) that exacerbate risk for cardiovascular disease. Social comparisons (i.e., self-evaluations relative to others) are known to influence PA in other groups, but their association in this population is unknown. The present study used ecological momentary assessment to examine this relation among women in midlife with hypertension or another CVD risk condition $\left(N=75, M_{B M I}=34.0 \mathrm{~kg} / \mathrm{m}^{2}\right)$. Participants completed 5 surveys per day and wore tri-axial accelerometers for 10 days. PA engagement was lower after women reported more comparisons than were typical for them (7-14\% reductions in PA for each additional comparison). These relations varied across days of observation and relations were positive on 34-58\% of days. Findings call for careful consideration of how best to harness any potential benefits of social comparison for promoting PA in this and other groups.
\end{abstract}

Keywords Physical activity - Midlife - Women's health · Social comparison

Danielle Arigo

arigo@ rowan.edu

Jacqueline A. Mogle

jam935@psu.edu

Joshua M. Smyth

jms1187@psu.edu

1 Department of Psychology, Rowan University, 201 Mullica Hill Road, Robinson Hall 116G, Glassboro, NJ 08028, USA

2 Department of Family Medicine, Rowan School of Osteopathic Medicine, Stratford, NJ, USA

3 Prevention Research Center, Pennsylvania State University, University Park, PA, USA

4 Departments of Biobehavioral Health and Medicine, Pennsylvania State University, University Park, PA, USA
During midlife (ages 40-60; Brim et al., 2019), women are at increased risk for cardiovascular disease due to biological factors consequent to aging, menopause, weight gain, and associated health conditions (e.g., hypertension; Kapoor et al., 2017). Women also decrease engagement in cardioprotective behaviors such as physical activity (PA) during this period (Davidson et al., 2010; Dugan et al., 2018). This decrease exacerbates the PA gender gap that exists throughout the lifespan (with men engaging in more PA than women; Appelman et al., 2015; Troiano et al., 2008) and further raises midlife women's cardiovascular risk. Promoting PA in this population could meaningfully reduce this risk (Jeong et al., 2019), though few existing interventions show long-term efficacy (Murray et al., 2017). Improving on the current understanding of barriers to and facilitators of PA among midlife women could point to novel intervention approaches for this population.

Consistent with several theories of health behavior, including social-cognitive theory (Bandura, 1998) and Midlife Women's Attitudes Toward Physical Activity Theory (MAPA; Im et al., 2010), perceptions of the social environment are key to PA engagement in this group. For example, more (vs. less) positive perceptions of social norms for PA are associated with greater PA engagement among women in midlife (Im et al., 2011, 2017; Janssen et al., 2014). Social comparisons, or self-evaluations relative to others (Festinger, 1954) represent a specific set of social perceptions that may have similar influences on midlife women's PA, but these relations have received little attention. Social comparisons are common in daily life and can be made across a range of dimensions (e.g., appearance, abilities, health behaviors). They can occur deliberately or automatically (Gilbert et al., 1995) and provide the comparer with information about their status in a given domain (Festinger, 1954). For example, comparing to someone perceived as 
"doing better" than the self (i.e., upward comparison) can demonstrate that improvement is possible and afford guidance for achieving similar outcomes (Bandura, 1998). Comparing to someone perceived as "doing worse" than the self (i.e., downward comparison) can provide satisfaction with one's own achievements and information about how to avoid undesirable outcomes (Wills, 1981). Comparing to someone perceived as "doing about the same" as the self (i.e., lateral comparisons) can show the comparer that they are keeping up with peers and thereby offer comfort (Helgeson \& Mickelson, 1995).

These expected benefits appear to serve as the rationale for including social comparison opportunities in PA interventions (i.e., exposure to information about other participants' PA engagement; Michie et al., 2011), and there is some evidence that comparison outperforms other social behavior change techniques such as support (e.g., Patel et al., 2016; Zhang et al., 2016). However, comparisons are not universally positive: upward comparisons can prompt dejection by underscoring the comparer's inferiority, and downward comparisons may trigger anxiety by illustrating an unavoidable future state (Buunk \& Ybema, 1997). Less is known about responses to lateral comparisons, though negative reactions to them also may be possible. Importantly, the vast majority of existing work on the consequences of social comparisons focus on self-reported outcomes, such as thoughts about engaging in PA (Arigo, Mogle, et al., 2020; Thøgersen-Ntoumani et al., 2018). It is unclear which, if any, types of comparisons are associated with changes in observable PA behavior. Additional information about relations between social comparison types and objectively assessed PA engagement is needed to fill this gap, to advance the present understanding of social comparison's role in everyday experiences and to define the circumstances under which comparisons might be appropriate or useful for PA intervention in at-risk groups such as women in midlife.

In particular, it is not clear whether any relation between comparison type and PA engagement represents stable differences between people versus fluctuation within the same person at different times (i.e., variability between- vs. within-persons). Between-person relations are described in the majority of existing work focused on social comparisons and health outcomes (e.g., from cross-sectional surveys or group-based randomized trials), and indicate for whom comparisons might be useful for increasing PA. In contrast, within-person relations can identify times when or contexts in which comparisons might be useful for this purpose (cf. Dunton, 2017; Smyth et al., 2017). Relations between social comparisons and PA at each level have distinct implications for social comparison as a target of PA interventions.

For example, knowing which women are likely to make comparisons that are negatively associated with subsequent PA (between-person) could help to target interventions toward women who would benefit from efforts to mitigate this association; such intervention may not be appropriate or useful for all women. In contrast, identifying the type(s) of comparison that are associated with less (vs. more) subsequent PA (within-person) could be useful for determining when or under what circumstances intervention skills or content would be most appropriate. Further, it is rare to see published work on social comparison that probes these effects by examining whether within-person relations with outcomes are consistent (vs. variable) across assessments, which could highlight the variety of potential behavioral responses (e.g., certain types of comparisons predicting greater and less PA engagement under different circumstances), and thus, the utility of a more nuanced approach to social comparisons in PA interventions.

Limited work using designs that capture within-person variation (e.g., ecological momentary assessment [EMA]; Smyth \& Stone, 2003) show that college women engage in less PA on days when they report experiencing (vs. not experiencing) a comparison of their health habits (across comparison directions; Arigo, Pasko, et al., 2020), though the likelihood of engaging in exercise to control weight is higher at and subsequent to times when they experience upward comparisons of appearance (Drutschinin et al., 2018). These findings indicate that young women can differentiate days and times when they do versus do not make comparisons, and that even when the comparison dimension is not PA, these distinct contexts are associated with PA outcomes at the within-person level. As noted, however, social comparisons are examined less often among women in midlife, particularly with respect to their relations with objectively assessed PA engagement. Extant evidence indicates that body comparisons are common in this age group and are associated with broader self-perceptions of appearance (which could motivate or hinder PA; Thompson \& BardoneCone, 2019; Watt \& Konnert, 2020), though comparisons in domains other than appearance have received little attention.

\section{Aims of the present study}

Social comparisons are proposed to predict health behaviors and vary within-person, though very little research has examined relations between comparisons and health behaviors at the within-person level (Arigo, Mogle, et al., 2020) or in atrisk groups such as women in midlife. To begin to address this gap, the overarching goal of this 10-day EMA study was to examine within-person relations between social comparisons and PA among midlife women with elevated risk for CVD - both overall, and the extent to which these relations differed across assessments. Specifically, the first aim of this study was to test for overall within-person relations between the number of social comparisons reported and subsequent PA 
engagement, using the total number of comparisons reported at each assessment and the frequency reported separately for each comparison direction. The second aim was to determine whether these relations varied within women across days. As existing work has shown both positive and negative consequences of comparisons, and as we expected these relations to vary across days, we did not have a priori hypotheses about the directions of these relations. Given that different intensities of PA have distinct implications for cardiovascular risk (Kokkinos \& Myers, 2010), outcomes were subsequent PA engagement in minutes of both light and moderate-to-vigorous PA. Engagement was assessed objectively in the $30 \mathrm{~min}$ after each survey was completed.

\section{Methods}

\section{Recruitment and participants}

Women were eligible to participate if they were between 40 and 60 years old (inclusive) and reported one or more of the following CVD risk factors: smoking (current or quit within the past three months), hypertension or prehypertension, prediabetes or type 2 diabetes, hyperlipidemia or hypercholesterolemia, or metabolic syndrome. Additional inclusion criteria required fluency in English, not currently pregnant, no comorbid medical conditions or psychiatric symptoms that would impede participation (e.g., injury, active psychosis), not currently engaged in weight loss or other behavior change efforts that might influence PA engagement, and access to a mobile device. Electronic advertisements appeared on community and news websites and were sent to students and employees at the supporting institutions. Active recruitment also took place in affiliated primary care offices; after chart review, eligible patients were given information about the study and invited to complete a brief telephone call to discuss their interest (see Fig. 1 for an enrollment flowchart). The final sample of 75 women $\left(M_{\text {Age }}=51.61\right)$ was 22\% Black, 2\% Latina, 1\% Asian-American, and 73\% White, with $3 \%$ identifying as mixed or other. The largest subsets of participants were married (56\%), identified as post-menopausal (41\%), and reported a diagnosis of hyperlipidemia or hypercholesterolemia (52\%). Average BMI was $34.02(S D=7.13)$ and the majority of participants had BMIs in the obese category (59\%). Additional demographic information can be found in Table 1 .

\section{Measures}

\section{Demographic information}

Height and weight were measured by research staff at participants' setup visits using a digital $\mathrm{Seca}^{\odot}$ scale and stadiometer. Measured height and weight were used to calculate
BMI $\left(\mathrm{kg} / \mathrm{m}^{2}\right)$. Other demographics such as race and menopause status were assessed with a baseline survey, completed before the initial setup appointment.

\section{Momentary survey: social comparison}

The wording of social comparison items was guided by the references below and refined through formative work with the population of interest (Arigo et al., 2021). At each survey, participants were asked whether they had made a how many times they made social comparisons since they woke up (first survey of the day) or in the last $3 \mathrm{~h}$ (all subsequent surveys of the day), using numeric text entry (Leahey et al., 2007). Follow-up items included what aspect(s) of the self they compared (e.g., appearance, health habits, abilities, etc.; Arigo, Pasko, et al., 2020; Wheeler \& Miyake, 1992) and the numbers of comparisons in each direction (i.e., people who seem to be doing better than I am [upward], people who seem to be doing about the same as I am [lateral], or people who seem to be doing worse than I am [downward]; numeric text entry for each; Leahey et al., 2007).

\section{Physical activity monitor}

PA was assessed using ActiGraph GT3X tri-axial accelerometers (ActriGraph Corp., Pensacola, FL), worn in alignment with the dominant hip during waking hours. PA parameters of interest were minutes of light, moderate, and vigorous activity for the $30 \mathrm{~min}$ following survey completion. This interval was selected to represent a proximal window of interest for both light PA and MVPA, though the effects reported below are consistent across 30-, 60, and 120-min intervals. Moderate and vigorous minutes were combined to estimate total minutes in moderate-to-vigorous intensity physical activity (MVPA). These values were calculated using the ActiPro package for RStudio (Dzubur, 2020). Nonwear was defined as periods with 60 or more continuous minutes without activity counts (i.e., activity counts $=0$; $\mathrm{cf}$. Pickering et al., 2016); these periods were excluded from analyses. MVPA cut points were those proposed by Matthews et al. (2008; see Arigo, Pasko, et al., 2020).

\section{Procedures}

The full protocol for this study is available (Arigo, Brown, et al., 2020a) and a summary is provided here. All procedures were approved by Institutional Review Boards at the supporting institutions; all data were collected between February 2019 and March 2020 (i.e., prior to the onset of the COVID-19 pandemic). Potential participants completed a screening call to confirm eligibility and schedule a setup appointment. These appointments included informed consent procedures and written documentation of consent, 
Fig. 1 CONSORT diagram

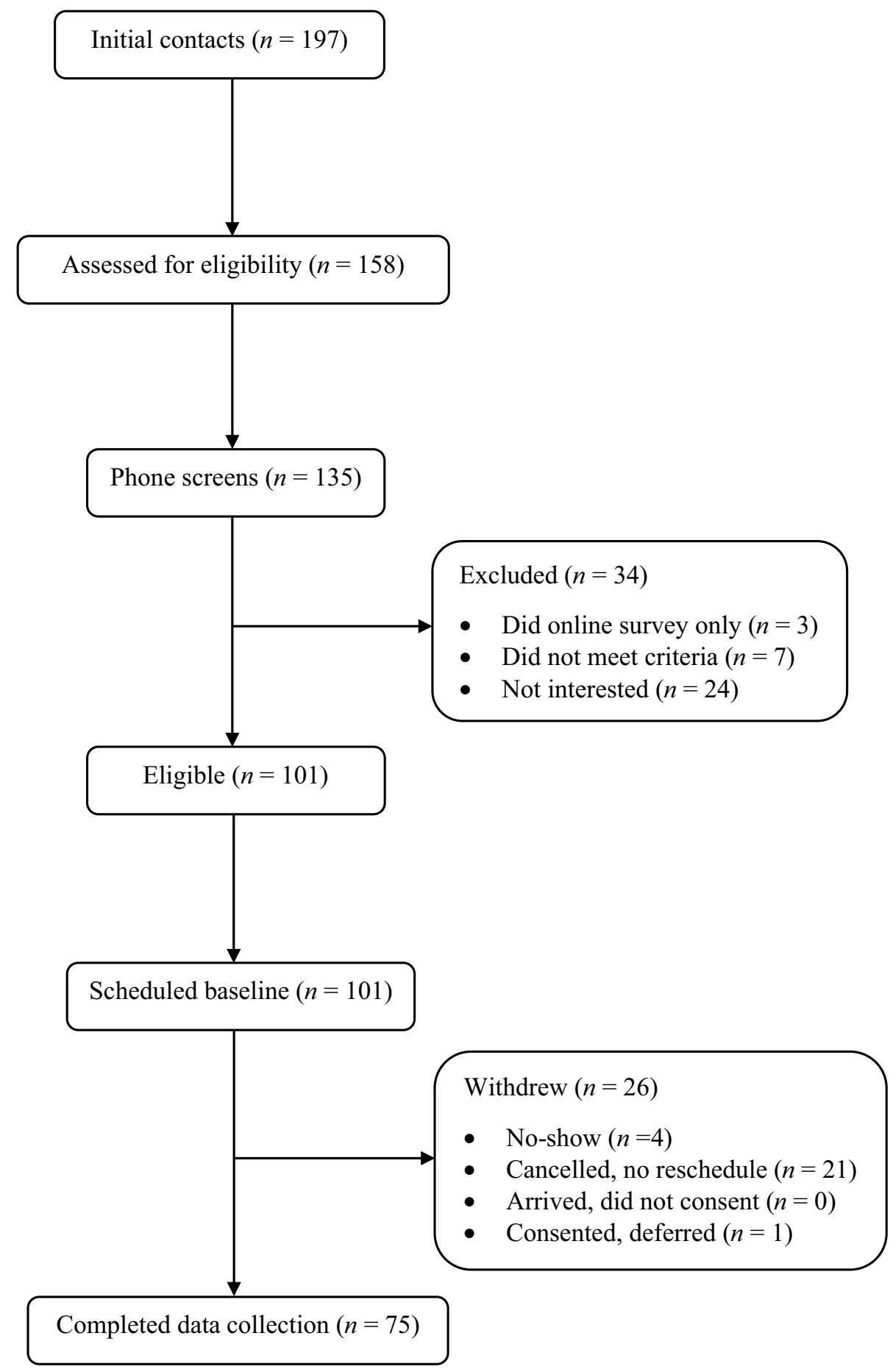

assessment of height and weight, introduction and testing of the accelerometer and momentary surveys, and instructions for identifying social comparisons. Participants were provided with examples of social comparisons and were encouraged to generate their own examples for discussion. At the end of the setup appointment, participants were scheduled for an exit interview to return the accelerometer and provide feedback on their experience. Staff then scheduled survey prompts to appear as text messages to the participant's smartphone, with embedded weblinks to each momentary survey. Responses were signal-contingent, such that participants reported on their experiences only in response to survey prompts. Surveys were scheduled around five semi-random times that fell between participants' wake and bed times, so that they did not appear at the same times each day; survey schedules did not differ between weekdays 
Table 1 Participant demographics $(n=75)$

\begin{tabular}{|c|c|c|c|}
\hline \multirow[b]{2}{*}{ Age } & \multicolumn{3}{|l|}{$M(S D)$} \\
\hline & $51.61(5.43)$ & & \\
\hline BMI & $34.02(7.13)$ & & \\
\hline Number of CVD risk factors & $1.63(0.82)$ & & \\
\hline Racial Identification & $n(\%)$ & Marital Status & $n(\%)$ \\
\hline Caucasian/White & $55(73 \%)$ & Never married & $12(16 \%)$ \\
\hline African American/Black & $16(22 \%)$ & Widowed & $4(5 \%)$ \\
\hline Asian or Pacific Islander & $1(1 \%)$ & Divorced & $11(15 \%)$ \\
\hline Hispanic/Latina & $2(3 \%)$ & Separated & $4(5 \%)$ \\
\hline Mixed/Other & $1(1 \%)$ & Married & $44(59 \%)$ \\
\hline Highest educational level & & Menopause Status & \\
\hline High School or GED & $7(9 \%)$ & Pre-menopause & $14(20 \%)$ \\
\hline Associate's degree, technical degree, or partial college & $16(21 \%)$ & Perimenopause & $16(23 \%)$ \\
\hline Bachelor's degree & $24(32 \%)$ & Post-menopause & $29(39 \%)$ \\
\hline Graduate/professional degree & $28(37 \%)$ & Other (e.g., surgical intervention) & $12(17 \%)$ \\
\hline$C V D$ risk condition $(s)$ & & Household Income & \\
\hline Hypercholesterolemia or hyperlipidemia & $39(52 \%)$ & $<\$ 25,000$ & $5(7 \%)$ \\
\hline Hypertension or prehyptertension & $35(47 \%)$ & $\$ 25,000-\$ 50,000$ & $12(16 \%)$ \\
\hline Type 2 diabetes & $30(40 \%)$ & $\$ 50,000-\$ 75,000$ & $12(16 \%)$ \\
\hline Metabolic syndrome & $8(11 \%)$ & $>\$ 75,000$ & $45(61 \%)$ \\
\hline Smoker (or quit in last 3 months) & $10(14 \%)$ & & \\
\hline
\end{tabular}

and weekends. Participants were asked to complete each survey within $1 \mathrm{~h}$ of receiving it. Participants received $\$ 15$ for attending the baseline appointment and $\$ 30$ when they returned for follow-up. Those who returned their accelerometers and completed more than $80 \%$ of EMA surveys received an additional $\$ 10$ bonus at follow-up.

\section{Statistical approach}

Sample size was calculated for within-person multilevel models (described below), following stimulations by Maas and Hox (2005). Using conservative estimates from previous studies and pilot work with the population of interest, a target sample size of 100 participants (level 3) with 10 days of observation (level 2) five times per day (level 1) exceeded thresholds for detecting within-person relations (level 1). Precautions against the spread of COVID-19 prevented recruitment and enrollment past March 2020, however. The final sample of 75 women with up to 50 observations each provided 3,750 possible observations and still afforded power $>0.80$ for within-person tests. Average completion across all surveys was $90 \%(S D=9.0 \%)$ and average completion in the designated 1-h time window was $80 \%$ $(S D=14.0 \%)$; only those surveys completed in this window were considered valid and included in analyses. Valid accelerometer data for the 30 min after valid surveys was available for 1482 observations.

\section{Missingness}

Missing data patterns were evaluated with bivariate correlations (percent of completed surveys) and SAS PROC GLIMMIX (binary variable indicating momentary survey completion). Missingness for surveys was positively associated with BMI $(r=0.24, p=0.03)$ and weekend days (versus weekdays; OR $=1.41,95 \%$ CI 1.12-1.78). Overall missingness was marginally associated with age $(r=0.21$, $p=0.07$ ), but was not related to other demographic characteristics $(p s>0.10)$ or to survey of the day or day in study $(0.92<$ ORs < 1.10). Missingness specific to social comparison items showed the same pattern. Valid accelerometer data were most likely to be missing after survey \#4 of the day (usually early evening; OR $=0.02,95 \%$ CI 0.01-0.03).

\section{Interrelations between predictors}

We examined whether at moments when a participant reported one type of social comparison, they were likely to also report other types. Correlations ranged from 0.23 to 0.68 with the highest correlation between upward and downward comparisons (all ps <0.01). As these correlations suggest moderate associations between related but distinct experiences, which is appropriate for the context, the number of each comparison was treated as an independent predictor of PA. Descriptive analyses of variability in predictors (numbers of comparisons overall, upward, lateral, and downward) used empty multilevel 
mixed models to estimate the proportion of variance accounted for at each level using the intraclass correlation coefficient (ICC).

\section{Primary analyses}

Multilevel models were used to address the nested structure of EMA data. Models included three levels to account for complex nested structure (surveys nested within days and days nested within persons); comparisons for each model showed significantly improved model fit with (vs. without) the day level ( $p s<0.01)$. Analyses were conducted in SAS 9.4 using a Poisson distribution (log link) in PROC GLIMMIX (SAS Institute Inc., Cary, NC) to address the non-normal distribution of PA engagement; all models employed likelihood approximation techniques to account for missingness (Laplace). Age, $\mathrm{BMI}$, weekday versus weekend, and survey of the day were included as covariates in all models.

For each continuous predictor of interest (i.e., overall number of comparisons and numbers of upward, lateral, and downward comparisons), variance was differentiated by calculating each person's mean across EMA responses (between-person) and the difference between this person mean and the response on a given survey (within-person; i.e., person-mean centering; Hoffman, 2015). This allows for interpretation of withinperson social comparisons as at times when a participant made more comparisons than their typical number, controlling for between-person means. Statistical significance for all fixed effects was set to $p<0.05$.

With respect to the second study aim (i.e., within-person variability in relations between comparisons and PA), models included random slopes for each predictor of interest. A significant random slope would indicate that relations between predictors and PA outcomes varied within women across assessment days. Model fit tests were conducted using the -2 loglikelihood (-2LL) to determine whether the random slope improved model fit over a model constraining slopes to be the same across participants. Differences between -2LL follow a chi-square distribution $\left(\chi^{2}\right)$ to determine whether the improvement was significant. For models where including the random slopes significantly improved fit, the plausible range of slopes and the percentage of days with positive slopes (indicating greater PA after social comparison reports) and negative slopes (indicating less PA after social comparison reports) were calculated, to aid in interpretation. Effect sizes are expressed as rate ratios indicating relative rates of $\mathrm{PA}$ engagement across different levels of the predictor.

\section{Results}

Across participants and moments, social comparisons were reported at $21 \%$ of surveys that were completed in the designated 1-h time window. Participants reported an average of 0.36 comparisons per survey $(S E=0.05)$ and the number of comparisons at a given survey ranged from 0 to 10. An $\mathrm{ICC}=0.20$ indicated that $20 \%$ of the variability in the overall number of comparisons reported was attributable to stable, between-person differences, whereas $80 \%$ was attributable to within-person fluctuation (and error). With respect to comparison direction, upward comparisons were most common (reported at $11 \%$ of all valid surveys), followed by lateral and downward comparisons ( $8 \%$ and $9 \%$ respectively). Between-person stability in the number of comparisons per direction was minimal (i.e., ICCs $<0.12$; see Table 2). Comparisons of abilities (e.g., skills, intelligence) were most common ( $9 \%$ of valid surveys), followed by comparisons of appearance $(7 \%)$ and emotional reactions (6\%; see Table 2$)$.

\section{Number of comparisons and PA engagement}

Participants had lower rates of light PA subsequent to reports with more comparisons than usual (overall; $p<0.001$, $\mathrm{RR}=0.93,95 \%$ CI $0.89-0.97$ ) and with reports of more upward or lateral comparisons than usual. Each additional upward comparison corresponded to a $14 \%$ lower rate of light PA $(p<0.001, \mathrm{RR}=0.86,95 \%$ CI $0.81-0.93)$ and each additional lateral comparison corresponded to a $7 \%$ lower rate of light PA $(p=0.02, \mathrm{RR}=0.93,95 \%$ CI $0.87-0.99$; see Table 3). Rates of both light PA and MVPA also were lower after surveys with more reported downward comparisons than usual, with each additional downward comparison corresponding to $14 \%$ lower rate of light PA $(p=0.002$; $\mathrm{RR}=0.86,95 \% \mathrm{CI} 0.78-0.95)$ and MVPA $(p=0.01$, $\mathrm{RR}=0.86$, 95\% CI 0.76-0.97; see Table 3). Rate of engagement in MVPA was not significantly associated the numbers of comparisons overall, upward, or lateral ( $p s>0.60)$.

\section{Variability in within-person relations}

Adding random slopes to the models predicting PA from the number of comparisons reported (i.e., allowing the relation between comparisons and PA to vary across days) significantly improved model fit (all $p s<0.01$ ). For example, the relation between overall number of comparisons and light PA $\left(\chi^{2}[1]=30.71\right)$ showed a plausible range of $95 \%$ slopes across days from -0.82 to $0.69(S D=0.37)$; this relation was negative for $56 \%$ of days and positive for $44 \%$. The plausible range of slopes for the relation between overall number of comparisons and MVPA $\left(\chi^{2}[1]=107.58\right)$ was -1.12 to 1.28 , with a negative relation on $45 \%$ of days and a positive relation on $55 \%$ (see Table 3 ). 
Table 2 Descriptive statistics for social comparison reports

\begin{tabular}{|c|c|c|c|c|}
\hline $\begin{array}{l}\text { Social comparison (Any Time in Report- } \\
\text { ing Window) }\end{array}$ & $n(\%)$ YES of all valid surveys & $\begin{array}{l}n(\%) Y E S \text { of surveys with } S C \\
\text { reported }\end{array}$ & $I C C$ & $B(S E)$ \\
\hline Any comparison & $617(21 \%)$ & & 0.20 & $0.36(0.05)$ \\
\hline Upward comparisons & $322(11 \%)$ & $322(52 \%)$ & 0.11 & $0.15(0.02)$ \\
\hline Lateral comparisons & $252(8 \%)$ & $252(41 \%)$ & 0.11 & $0.14(0.02)$ \\
\hline Downward comparisons & $261(9 \%)$ & $261(42 \%)$ & 0.07 & $0.08(0.01)$ \\
\hline Occurrence by type & \multicolumn{3}{|c|}{ Any time in window $n(\%)$} & Most recent $n(\%)$ \\
\hline Upward & \multicolumn{2}{|c|}{$322(11 \%)$} & & $234(43 \%)$ \\
\hline Lateral & \multicolumn{2}{|c|}{$252(8 \%)$} & & $164(30 \%)$ \\
\hline Downward & \multicolumn{2}{|c|}{$261(9 \%)$} & & $145(27 \%)$ \\
\hline Appearance & \multicolumn{2}{|c|}{$197(7 \%)$} & & $82(17 \%)$ \\
\hline Health habits & \multicolumn{2}{|c|}{$155(5 \%)$} & & $51(11 \%)$ \\
\hline Status & \multicolumn{2}{|c|}{$166(6 \%)$} & & $51(11 \%)$ \\
\hline Emotion regulation & \multicolumn{2}{|c|}{$181(6 \%)$} & & $79(17 \%)$ \\
\hline Personality & \multicolumn{2}{|c|}{$140(5 \%)$} & & $35(7 \%)$ \\
\hline Abilities & \multicolumn{2}{|c|}{$279(9 \%)$} & & $135(28 \%)$ \\
\hline Other & \multicolumn{2}{|c|}{$54(2 \%)$} & & $44(9 \%)$ \\
\hline
\end{tabular}

$n(\%)$ yes indicates report of comparison occurrence, out of 2967 valid surveys (any time window) and 617 surveys where comparisons were reported (most recent comparison). $\mathrm{ICC}=$ intraclass correlation coefficient

Relations between the number of comparisons in each direction and PA showed similar patterns, though the proportion of days with negative slopes varied across direction of comparison. For relations between number of upward comparisons and light PA $\left(\chi^{2}[1]=11.48\right)$ and MVPA $\left(\chi^{2}[1]=64.16\right)$, the plausible ranges of slopes across days were -0.83 to 0.67 ( $S D=0.37$; negative for $58 \%$ of days) for light PA and -1.08 to 1.32 ( $S D=0.60$; negative for $42 \%$ of days) for MVPA, respectively. For relations between number of lateral comparisons and light PA $\left(\chi^{2}[1]=7.93\right)$ and MVPA $\left(\chi^{2}[1]=26.89\right)$, the plausible ranges of slopes across days were -0.76 to 0.68 ( $S D=0.36$; negative for $54 \%$ of days) for light PA and -1.08 to 1.23 ( $S D=0.59$; negative for $43 \%$ of days) for MVPA, respectively. For relations between number of downward comparisons and light PA $\left(\chi^{2}[1]=9.30\right)$ and MVPA $\left(\chi^{2}[1]=21.85\right)$, the plausible ranges of slopes across days were -0.90 to $0.60(S D=0.37$; negative for $66 \%$ of days) and -1.37 to 1.07 ( $S D=0.62$; negative for $60 \%$ of days), respectively.

\section{Discussion}

Understanding psychosocial determinants of PA in midlife women's daily lives could help to improve PA promotion efforts for this population. Toward this goal, the present study is the first to identify social comparison as a predictor of PA engagement in this population and to describe this relation at the within-person level. In addition, as it is one of the few studies of relations between social comparison and objectively assessed PA more broadly, the present findings also offer important contributions to our understanding of basic social comparison processes and their implications for daily experiences.

Because comparisons can happen without full awareness and may be perceived as socially undesirable (Helgeson \& Taylor, 1993), it can be difficult to estimate their frequency in the natural environment and accurately capture individual differences and within-person variability. EMA and other intensive assessment methods can reduce retrospective recall biases and improve the accuracy of these estimates (Smyth \& Stone, 2003), though little existing research explicitly describes variability at different levels (Arigo, Mogle, et al., 2020). Findings from the present study indicate that comparisons occurred approximately twice per day, on average, and that the majority of variability in their frequency was within-person. Thus, although some women may consistently experience more comparisons than others, the frequency of comparison experiences varies widely for individual women.

Specifically, overall within-person relations between the number of social comparisons and subsequent PA were negative: at times when women experienced more comparisons than usual, they were $7 \%-14 \%$ less active in the following 30 min for each additional comparison. This pattern held across upward, downward, and lateral comparisons, particularly for light PA, suggesting that naturally occurring comparisons may be detrimental (rather than beneficial) for midlife women's subsequent PA. Importantly, however, adding a random slope improved model fit for each of these within-person 
Table 3 Multilevel model estimates for tests of relations between social comparison and physical activity engagement 30 min after each survey

\begin{tabular}{|c|c|c|}
\hline & Minutes of light activity & Minutes of MVPA \\
\hline Total number of comparisons & $B(S E)$ & $B(S E)$ \\
\hline Intercept & $1.56(0.06)^{* *}$ & $1.19(0.08)^{* *}$ \\
\hline BMI & $0.002(0.006)$ & $0.0007(0.007)$ \\
\hline Age & $0.004(0.007)$ & $-0.004(0.009)$ \\
\hline Weekend vs. weekday & $0.76(0.05)$ & $-0.03(0.07)$ \\
\hline Survey of the day & $-0.21(0.02)^{* *}$ & $-0.25(-0.02)^{* *}$ \\
\hline Between-person effect & $-0.11(0.10)$ & $-0.04(0.13)$ \\
\hline \multirow[t]{2}{*}{ Within-person effect } & $-0.07(0.02)^{* *}$ & $0.005(0.03)$ \\
\hline & Range of Slopes (\% Positive) & Range of Slopes (\% Positive) \\
\hline Random effects & $-0.82-0.69(44 \%)$ & $-1.12-1.28(55 \%)$ \\
\hline Number of upward comparisons & $B(S E)$ & $B(S E)$ \\
\hline Intercept & $1.57(0.06)^{* *}$ & $1.19(0.08)^{* *}$ \\
\hline BMI & $0.002(0.006)$ & $0.001(0.007)$ \\
\hline Age & $0.003(0.007)$ & $-0.005(0.009)$ \\
\hline Weekend vs. weekday & $0.08(0.05)$ & $-0.02(0.07)$ \\
\hline Survey of the day & $-0.21(0.02)^{* *}$ & $-0.25(0.02)^{* *}$ \\
\hline Between-person effect & $0.012(0.01)$ & $-0.02(0.13)$ \\
\hline \multirow[t]{2}{*}{ Within-person effect } & $-0.15(0.03)^{* *}$ & $0.02(0.04)$ \\
\hline & Range of Slopes (\% Positive) & Range of Slopes (\% Positive) \\
\hline Random effects & $-0.83-0.67(42 \%)$ & $-1.08-1.32(58 \%)$ \\
\hline Number of lateral comparisons & $B(S E)$ & $B(S E)$ \\
\hline Intercept & $1.57(0.06)^{* *}$ & $1.19(0.08)^{* *}$ \\
\hline BMI & $0.002(0.006)$ & $0.001(0.007)$ \\
\hline Age & $0.004(0.007)$ & $-0.005(0.009)$ \\
\hline Weekend vs. weekday & $0.07(0.05)$ & $-0.02(0.07)$ \\
\hline Survey of the day & $-0.21(0.02)^{* *}$ & $-0.25(0.02)^{* *}$ \\
\hline Between-person effect & $-0.12(0.10)$ & $-0.02(0.13)$ \\
\hline \multirow[t]{2}{*}{ Within-person effect } & $-0.08(0.03)^{*}$ & $-0.02(0.04)$ \\
\hline & Range of Slopes (\% Positive) & Range of Slopes (\% Positive) \\
\hline Random effects & $-0.76-0.68(46 \%)$ & $-1.08-1.23(57 \%)$ \\
\hline Number of downward comparisons & $B(S E)$ & $B(S E)$ \\
\hline Intercept & $1.57(0.06)^{* *}$ & $1.16(0.08)^{* *}$ \\
\hline BMI & $0.002(0.006)$ & $0.0005(0.007)$ \\
\hline Age & $0.004(0.007)$ & $-0.006(0.009)$ \\
\hline Weekend vs. weekday & $0.08(0.05)$ & $-0.01(0.07)$ \\
\hline Survey of the day & $0.21(0.02)^{* *}$ & $-0.24(0.02)^{* *}$ \\
\hline Between-person effect & $-0.10(0.10)$ & $-0.02(0.13)$ \\
\hline \multirow[t]{2}{*}{ Within-person effect } & $-0.15(0.05)^{* *}$ & $-0.15(0.06)^{*}$ \\
\hline & Range of Slopes (\% Positive) & Range of Slopes (\% Positive) \\
\hline Random effects & $-0.90-0.60(34 \%)$ & $-1.37-1.07(40 \%)$ \\
\hline
\end{tabular}

${ }^{*} p<0.05, * * p<0.01$

relations, indicating that their strength-and importantly, direction-varied within women between days. On $34 \%$ to $58 \%$ of days, women engaged in more PA, rather than less, after experiencing more comparisons than usual, and positive associations occurred more frequently for MVPA than light PA. Thus, experiencing additional comparisons may facilitate PA on some days and impede it on others. As this is a novel observation, additional research is needed to first determine the daily circumstances associated with positive versus negative relations between social comparisons and PA.

For example, the daily variability in relations between comparisons and PA may reflect shifts in women's deeper processing of their comparisons, such as identification with 
versus contrast against their comparison targets. According to the Identification/Contrast Model (Buunk \& Ybema, 1997), downward comparison prompts negative affect via a focus on similarities (i.e., identification, showing that an undesirable state is present or likely to occur for the self) and positive affect via a focus on differences (i.e., contrast, emphasizing the distance between current and undesired circumstances). Conversely, upward comparison prompts negative affect via contrast (as this increases the salience of the comparer's inferiority and distance from desired goals) and positive affect via identification (as the comparer's goals seem achievable; see Arigo et al., 2015; Buunk \& Dijkstra, 2017). Subsequent PA engagement may follow a similar pattern; for example, PA may be greater on days when women have positive responses to additional downward comparisons (contrast) and lower on days when women have negative responses to additional downward comparisons (identification).

As negative responses to comparisons also can motivate behavior change (e.g., Mahler, 2018), however, it is unlikely that one type of response to additional comparisons consistently explains daily variation in their relations with women's PA. It is possible that positive responses to additional comparisons predict greater subsequent PA on some days and less subsequent PA on others, and similarly for negative responses. Existing research shows betweenperson differences in identification/contrast processes (Van der Zee et al., 2000) but has not examined within-person variability (Arigo, Mogle, et al., 2020), and lateral comparisons have received little attention with respect to this model. Additional insight into this aspect of variability in relations between comparisons and PA could provide critical information about the contexts in which comparisons are useful for PA promotion and point to their mechanisms of action among women in midlife (and potentially, more broadly).

\section{Implications and future directions}

The present study is one of the first in a series of steps necessary to fully understand relations between experiences of social comparisons and PA. For example, participants in this study were asked to report on their comparisons over the preceding 3-h time frame. Consequently, the specific timing of comparisons during these windows is unknown, as is whether they were engaged in PA at the times that their comparisons occurred. Additional work is needed to further clarify the temporal sequencing of naturally occurring comparisons and PA, as relations between these experiences may be even more complex than described here (e.g., on some days, making comparisons while active early in the day leads to additional comparisons and/or PA later in the day). Event-contingent recording allows participants to report on an experience such as comparison immediately after it occurs; this could be useful for improving the temporal specificity of comparisons, either alone or in tandem with signal-contingent recording (used in the present study; cf. Smith et al., 2020).

Another critical step in this line of work is to determine whether the observed within-person relations are causal and responsive to intervention. With respect to causality, experimental exposure to distinct comparison targets shows between-person differences in affect and outcomes such as desire for affiliation (Derlega et al., 2008; Gerber et al., 2018; Wood et al., 1985), as well as in reports of exercise motivation (Diel \& Hofmann, 2019). As noted, existing literature also indicates that social comparison is used as a behavior change technique in many PA interventions (Bélanger-Gravel et al., 2011; Howlett et al., 2019), particularly in digital programs (e.g., smartphone applications; Arigo, Brown, et al., 2020b; Conroy et al., 2014), and that comparison can be an effective mechanism of PA behavior change (though it is captured between-person; Olander et al., 2013; Patel et al., 2016; Zhang et al., 2016).

Yet, the present findings highlight the possibility that there are times when social comparisons, and potentially, comparison-based intervention, may be more harmful than helpful. As little existing intervention work has examined experimental effects within-person, however, the optimal level of intervention (if one exists) has not been identified. Future work using intensive assessment designs could marry these related but independent lines of inquiry. For example, ecological momentary intervention (EMI) uses prompts during daily life to experimentally expose participants to stimuli, to assess causal relations in the natural environment (Heron \& Smyth, 2010). A protocol to test the within-person effects of comparisons on PA could provide comparison exposure (e.g., to an upward PA target) via smartphone and assess subsequent changes in PA, as well as proposed mediators of this relation (e.g., identification, affective response) and contextual moderators (e.g., day of the week, days with vs. without experiences such as work stress). Similarly, a test of within-person intervention effects might provide guidance toward particular types of engagement with targets (e.g., contrast against a downward target) via smartphone and assess (1) to what extent this guidance was followed, and (2) subsequent changes in PA.

Together, such work would allow for investigation of mechanistic pathways between social comparison processes and PA outcomes and potential intervention effects, as well as differences in each by context, to identify timesensitive methods of harnessing social comparison to promote PA. Although such steps are likely to have broad applications, this work may be especially relevant for PA promotion among midlife women, who cite lack of PA role models as a barrier to PA engagement (Cannioto, 2010) 
and show particular interest in peer models of PA engagement as an intervention technique (Rowland et al., 2018).

\section{Strengths and limitations}

Strengths of this study include recruitment of an at-risk clinical sample, objective assessment of PA, and use of a preregistered EMA design to examine within-person relations between experiences of interest. Survey completion and PA monitor wear also were on par with or better than typical rates for EMA (cf. Maher et al., 2017) and were impressive for 10 days of assessment. However, precautions against the spread of COVID-19 resulted in a smaller sample size than intended, and despite efforts to recruit a diverse sample, majority of participants were Caucasian and highly educated. The 30-min time window selected for relations with objectively assessed PA was one of many that might be of interest (cf. Kim et al., 2020; Maher et al., 2018; Pickering et al., 2016), though we note that the observed relations were consistent across 30-, 60-, and 120-min windows.

Further, as indicated, the design of the present study did not focus on assessment of comparison at the time of its occurrence, and even women's most recent comparisons could have been any time in the 3 -h reporting window. Consequently, the observed relations between social comparisons and PA outcomes may be specific to the act of reflecting and reporting on comparisons, which may have increased their salience at the moment of survey completion. Additional work using larger, more diverse samples and alternative designs (e.g., reporting at the time of the comparison event) are needed to clarify the boundaries of relations observed in the current study. As the present study focused on a narrow population with elevated CVD risk, an important aspect of future research will be to determine whether the relations observed here generalize to other populations or objectively assessed behaviors.

\section{Conclusions}

The experience of social comparison is common among women in midlife and may represent a useful target of PA intervention for this population. Importantly, although social comparison is recognized as an effective mechanism of PA behavior change (Olander et al., 2013), there is little evidence that existing interventions with social comparison components address individual or contextual differences in comparison consequences for PA (cf. Arigo, Brown, et al., $2020 \mathrm{~b}$ ). Findings from the present study show that, overall, experiencing more (vs. fewer) comparisons than usual is associated with less subsequent PA engagement among women in midlife, and thus, highlights the overall need for nuanced approaches to social comparison as a technique to promote PA behavior change in this population. Given that women engaged in more PA after experiencing additional comparisons on $34-58 \%$ of days, however, it will be critical to further determine when (and what types of) social comparisons may be helpful versus harmful for PA among women in midlife and other at-risk groups.

Acknowledgements The authors would like to thank M. Cole Ainsworth, Ph.D., Megan M. Brown, B.S., Kristen Pasko, M.A., Laura Travers, M.S., and Emily Vendetta, B.A. for their assistance with data collection and management.

Authors' contributions All authors contributed to study design and manuscript preparation. DA oversaw data collection and management. JAM conducted statistical analyses.

Data availability Data are available from the first author upon reasonable request.

Funding This work was supported by the National Heart, Lung, and Blood Institute (K23 HL136657, PI: Arigo).

\section{Declarations}

Conflict of interest The authors declare there is no conflict of interest.

Consent to participate Procedures were approved by the institutional review boards at Rowan University and Rowan School of Osteopathic Medicine.

Informed consent Rowan University and Rowan School of Osteopathic Medicine. All participants provided written informed consent.

\section{References}

Appelman, Y., van Rijn, B. B., Monique, E., Boersma, E., \& Peters, S. A. (2015). Sex differences in cardiovascular risk factors and disease prevention. Atherosclerosis, 241(1), 211-218

Arigo, D., Brown, M. M., Pasko, K., Ainsworth, M. C., Travers, L., Gupta, A. A., Symons Downs, D., \& Smyth, J. M. (2020a). Rationale and design of the women's health and daily experiences project: Protocol for an ecological momentary assessment study to identify real-time predictors of midlife women's physical activity. JMIR Research Protocols, 9(10), e19044.

Arigo, D., Brown, M. M., Pasko, K., \& Suls, J. (2020b). Social comparison features in physical activity promotion apps: Scoping metareview. Journal of Medical Internet Research, 22(3), e15642.

Arigo, D., Mogle, J. A., Brown, M. M., Pasko, K., Travers, L., Sweeder, L., \& Smyth, J. M. (2020). Methods to assess social comparison processes within persons in daily life: A scoping review. Frontiers in Psychology, 10, 1-21

Arigo, D., Mogle, J. A., Brown, M. M., \& Gupta, A. (2021). A multistudy approach to refining ecological momentary assessment measures for use among midlife women with elevated risk for cardiovascular disease. mHealth. https://doi.org/10.21037/mheal th-20-143

Arigo, D., Pasko, K., \& Mogle, J. A. (2020). Daily relations between social perceptions and physical activity among college women. Psychology of Sport and Exercise, 47, 101528. 
Arigo, D., Suls, J. M., \& Smyth, J. M. (2015). Social comparisons and chronic illness: Research synthesis and clinical implications. Health Psychology Review, 8(2), 154-214

Bandura, A. (1998). Health promotion from the perspective of social cognitive theory. Psychology \& Health, 13(4), 623-649

Bélanger-Gravel, A., Godin, G., Vézina-Im, L. A., Amireault, S., \& Poirier, P. (2011). The effect of theory-based interventions on physical activity participation among overweight/obese individuals: A systematic review. Obesity Reviews, 12(6), 430-439

Brim, O. G., Ryff, C. D., \& Kessler, R. C. (2019). How healthy are we?: A national study of well-being at midlife. University of Chicago Press.

Buunk, A. P., \& Dijkstra, P. (2017). Social comparisons and wellbeing. In M. Robinson \& M. Eid (Eds.), The happy mind: Cognitive contributions to well-being. (pp. 311-330). Springer.

Buunk, B. P., \& Ybema, J. F. (1997). Social comparisons and occupational stress: The identification-contrast model. In Buunk, B. P., \& Gibbons, F. X. (Eds.), Health, coping, and well-being: Perspectives from Social Comparison Theory (pp. 359-388). Lawrence Erlbaum Associates, Inc.

Cannioto, R. A. (2010). Physical activity barriers, behaviors, and beliefs of overweight and obese working women: A preliminary analysis. Women in Sport \& Physical Activity Journal; Greensboro, 19(1), 70-85

Conroy, D. E., Yang, C. H., \& Maher, J. P. (2014). Behavior change techniques in top-ranked mobile apps for physical activity. American Journal of Preventive Medicine, 46(6), 649-652

Davidson, L. E., Tucker, L., \& Peterson, T. (2010). Physical activity changes predict abdominal fat change in midlife women. Journal of Physical Activity and Health, 7(3), 316-322

Derlega, V. J., Greene, K., Henson, J. M., \& Winstead, B. A. (2008). Social comparison activity in coping with HIV. International Journal of STD \& AIDS, 19(3), 164-167

Diel, K., \& Hofmann, W. (2019). Inspired to perspire: The interplay of social comparison direction and standard extremity in the context of challenging exercising goals. Social Cognition, $37(3), 247-265$

Drutschinin, K., Fuller-Tyszkiewicz, M., Paoli, T. D., Lewis, V., \& Krug, I. (2018). The daily frequency, type, and effects of appearance comparisons on disordered eating. Psychology of Women Quarterly, 42(2), 151-161

Dugan, S. A., Gabriel, K. P., Lange-Maia, B. S., \& Karvonen-Gutierrez, C. (2018). Physical activity and physical function: Moving and aging. Obstetrics and Gynecology Clinics, 45(4), 723-736

Dunton, G. F. (2017). Ecological momentary assessment in physical activity research. Exercise and Sport Sciences Reviews, 45(1), 48-54

Dzubur, E. (2020). Eldinidle/ActiPro [R]. GitHub. Available at https:// github.com/eldinidle/ActiPro. Accessibility verified March 11, 2021.

Festinger, L. (1954). A theory of social comparison processes. Human Relations, 7(2), 117-140

Gerber, J. P., Wheeler, L., \& Suls, J. (2018). A social comparison theory meta-analysis $60+$ years on. Psychological Bulletin, 144(2), 177-197

Gilbert, D. T., Giesler, R. B., \& Morris, K. A. (1995). When comparisons arise. Journal of Personality and Social Psychology, 69(2), $227-236$

Helgeson, V. S., \& Mickelson, K. D. (1995). Motives for social comparison. Personality and Social Psychology Bulletin, 21(11), 1200-1209

Helgeson, V. S., \& Taylor, S. E. (1993). Social comparisons and adjustment among cardiac patients 1. Journal of Applied Social Psychology, 23(15), 1171-1195

Heron, K. E., \& Smyth, J. M. (2010). Ecological momentary interventions: Incorporating mobile technology into psychosocial and health behaviour treatments. British Journal of Health Psychology, 15(1), 1-39

Hoffman, L. (2015). Longitudinal analysis: Modeling within-person fluctuation and change. Routledge.

Howlett, N., Trivedi, D., Troop, N. A., \& Chater, A. M. (2019). Are physical activity interventions for healthy inactive adults effective in promoting behavior change and maintenance, and which behavior change techniques are effective? A systematic review and meta-analysis. Translational Behavioral Medicine, 9(1), 147-157

Im, E. O., Ko, Y., Chee, E., Chee, W., \& Mao, J. J. (2017). Clusters of midlife women by physical activity and their racial/ethnic differences. Menopause, 24(4), 417-425

Im, E. O., Lee, B., Chee, W., \& Stuifbergen, A. (2011). Attitudes toward physical activity of white midlife women. Journal of Obstetric, Gynecologic \& Neonatal Nursing, 40(3), 312-321

Im, E. O., Stuifbergen, A. K., \& Walker, L. (2010). A situation-specific theory of midlife women's attitudes toward physical activity (MAPA). Nursing Outlook, 58(1), 52-58

Janssen, I., Dugan, S. A., Karavolos, K., Lynch, E. B., \& Powell, L. H. (2014). Correlates of 15-year maintenance of physical activity in middle-aged women. International Journal of Behavioral Medicine, 21(3), 511-518

Jeong, S. W., Kim, S. H., Kang, S. H., Kim, H. J., Yoon, C. H., Youn, T. J., \& Chae, I. H. (2019). Mortality reduction with physical activity in patients with and without cardiovascular disease. European Heart Journal, 40(43), 3547-3555

Kapoor, E., Collazo-Clavell, M. L., \& Faubion, S. S. (2017). Weight gain in women at midlife: A concise review of the pathophysiology and strategies for management. Mayo Clinic Proceedings, 92(10), 1552-1558

Kim, J., Conroy, D. E., \& Smyth, J. M. (2020). Bidirectional associations of momentary affect with physical activity and sedentary behaviors in working adults. Annals of Behavioral Medicine, 54(4), 268-279

Kokkinos, P., \& Myers, J. (2010). Exercise and physical activity: Clinical outcomes and applications. Circulation, 122(16), 1637-1648

Leahey, T. M., Crowther, J. H., \& Mickelson, K. D. (2007). The frequency, nature, and effects of naturally occurring appearancefocused social comparisons. Behavior Therapy, 38(2), 132-143

Maas, C., \& Hox, J. (2005). Sufficient sample sizes for multilevel modeling. Methodology, 1(3), 86-92

Maher, J. P., Rebar, A. L., \& Dunton, G. F. (2018). Ecological momentary assessment is a feasible and valid methodological tool to measure older adults' physical activity and sedentary behavior. Frontiers in Psychology, 9, 1-11

Maher, J. P., Rhodes, R. E., Dzubur, E., Huh, J., Intille, S., \& Dunton, G. F. (2017). Momentary assessment of physical activity intention-behavior coupling in adults. Translational Behavioral Medicine, 7(4), 709-718

Mahler, H. I. (2018). The relative role of cognitive and emotional reactions in mediating the effects of a social comparison sun protection intervention. Psychology \& Health, 33(2), 235-257

Matthews, C. E., Chen, K. Y., Freedson, P. S., Buchowski, M. S., Beech, B. M., Pate, R. R., \& Troiano, R. P. (2008). Amount of time spent in sedentary behaviors in the United States, 20032004. American Journal of Epidemiology, 167(7), 875-881

Michie, S., Ashford, S., Sniehotta, F. F., Dombrowski, S. U., Bishop, A., \& French, D. P. (2011). A refined taxonomy of behaviour change techniques to help people change their physical activity and healthy eating behaviours: The CALO-RE taxonomy. Psychology \& Health, 26(11), 1479-1498

Mollee, J. S., \& Klein, M. C. (2016). The effectiveness of upward and downward social comparison of physical activity in an online intervention. 2016 15th International Conference on Ubiquitous Computing and Communications and 2016 International Symposium on Cyberspace and Security (IUCC-CSS), 109-115. 
Murray, J. M., Brennan, S. F., French, D. P., Patterson, C. C., Kee, F., \& Hunter, R. F. (2017). Effectiveness of physical activity interventions in achieving behaviour change maintenance in young and middle aged adults: A systematic review and meta-analysis. Social Science \& Medicine, 192, 125-133

Olander, E. K., Fletcher, H., Williams, S., Atkinson, L., Turner, A., \& French, D. P. (2013). What are the most effective techniques in changing obese individuals' physical activity self-efficacy and behaviour: A systematic review and meta-analysis. International Journal of Behavioral Nutrition and Physical Activity, 10(1), 1-15

Patel, M. S., Volpp, K. G., Rosin, R., Bellamy, S. L., Small, D. S., Fletcher, M. A., Osman-Koss, R., Brady, J. L., Haff, N., \& Lee, S. M. (2016). A randomized trial of social comparison feedback and financial incentives to increase physical activity. American Journal of Health Promotion, 30(6), 416-424

Pickering, T. A., Huh, J., Intille, S., Liao, Y., Pentz, M. A., \& Dunton, G. F. (2016). Physical activity and variation in momentary behavioral cognitions: An ecological momentary assessment study. Journal of Physical Activity and Health, 13(3), 344-351

Pila, E., Barlow, M. A., Wrosch, C., \& Sabiston, C. M. (2016). Comparing the body to superior others: Associations with daily exercise and body evaluation in men and women. Psychology of Sport and Exercise, 27, 120-127

Rowland, S. A., Cohen, M. Z., Pullen, C. H., Schulz, P. S., Berg, K. E., \& Yates, B. C. (2018). Perceptions of a peer modeling workplace physical activity intervention for women. Workplace Health \& Safety, 66(9), 437-443

Smith, K. E., Mason, T. B., Juarascio, A., Weinbach, N., Dvorak, R., Crosby, R. D., \& Wonderlich, S. A. (2020). The momentary interplay of affect, attention bias, and expectancies as predictors of binge eating in the natural environment. International Journal of Eating Disorders, 53(4), 586-594

Smyth, J. M., Juth, V., Ma, J., \& Sliwinski, M. (2017). A slice of life: Ecologically valid methods for research on social relationships and health across the life span. Social and Personality Psychology Compass, 11(10), e12356

Smyth, J. M., \& Stone, A. A. (2003). Ecological momentary assessment research in behavioral medicine. Journal of Happiness Studies, 4(1), $35-52$
Thøgersen-Ntoumani, C., Ntoumanis, N., Carey, R., Dodos, L., Quested, E. J., \& Chatzisarantis, N. (2018). A diary study of appearance social comparisons and need frustration in young women. Personality and Individual Differences, 122, 120-126

Thompson, K. A., \& Bardone-Cone, A. M. (2019). Evaluating attitudes about aging and body comparison as moderators of the relationship between menopausal status and disordered eating and body image concerns among middle-aged women. Maturitas, 124, 25-31

Troiano, R. P., Berrigan, D., Dodd, K. W., Masse, L. C., Tilert, T., \& Mcdowell, M. (2008). Physical activity in the United States measured by accelerometer. Medicine \& Science in Sports \& Exercise, 40(1), 181-188

Van der Zee, K., Buunk, B., Sanderman, R., Botke, G., \& Van den Bergh, F. (2000). Social comparison and coping with cancer treatment. Personality and Individual Differences, 28(1), 17-34

Watt, A. D., \& Konnert, C. A. (2020). Body satisfaction and selfesteem among middle-aged and older women: The mediating roles of social and temporal comparisons and self-objectification. Aging \& Mental Health, 24(5), 797-804

Wheeler, L., \& Miyake, K. (1992). Social comparison in everyday life. Journal of Personality and Social Psychology, 62(5), 760-773

Wheeler, L., \& Reis, H. T. (1991). Self-recording of everyday life events: Origins, types, and uses. Journal of Personality, 59(3), 339-354

Wills, T. A. (1981). Downward comparison principles in social psychology. Psychological Bulletin, 90(2), 245-271

Wood, J. V., Taylor, S. E., \& Lichtman, R. R. (1985). Social comparison in adjustment to breast cancer. Journal of Personality and Social Psychology, 49(5), 1169-1183

Zhang, J., Brackbill, D., Yang, S., Becker, J., Herbert, N., \& Centola, D. (2016). Support or competition? How online social networks increase physical activity: A randomized controlled trial. Preventive Medicine Reports, 4, 453-458

Publisher's Note Springer Nature remains neutral with regard to jurisdictional claims in published maps and institutional affiliations. 